\title{
Reliable and Efficient Data Transfer in Wireless Sensor Networks via Out-of-Sequence Forwarding and Delayed Request for Missing Packets
}

\author{
Damayanti Datta and Sukhamay Kundu \\ Computer Science Dept, Louisiana State University \\ Baton Rouge, LA 70803, USA \\ \{ddattal@lsu.edu,kundu@csc.lsu.edu\}
}

\begin{abstract}
We present a new protocol for lossless data transfer in wireless sensor networks using less time and fewer messages in comparison to the well-known protocol $P S F Q$ [1], without compromising the reliability. The two key features for the better performance of our protocol are out-of-sequence packet forwarding and delayed request for missing packets.
\end{abstract}

\section{Introduction}

Many applications in wireless sensor networks require reliable data transfer, e.g., instructions sent from a sink to a set of destination nodes to execute a new task. We typically partition the entire data set into a number of (bounded) fixed size packets $p_{i}, 0 \leq i<n$ $(n \geq 1)$ and then send them packet by packet. The detection and recovery of missing packets are performed at the destination nodes and also at the intermediate nodes; the latter can reduce the total delivery time of all packets to the destination nodes and also decrease the number of messages. The reduction of number of messages is important due to the energy constraints of the sensors and the reduction of delivery time is important for time-critical applications.

A well-known transport layer reliable data transfer protocol, with near-zero tolerance for data loss, is Pump Slowly Fetch Quickly (PSFQ) [1]. It is a nonacknowledgment based method and uses in-sequence (IS) forwarding, where a node $x$ sends a packet $p_{j}$ only if it has previously sent each packet $p_{i}, i<j$, at least once. This tends to delay the delivery of $p_{j}$ to a node and to increase the total delivery time. The Reliable Multi-Segment Transport protocol (RMST) [2] implements reliability at both MAC and transport layer, using a PSFQ-like method for the latter.

Our protocol is also non-acknowledgment based, but it uses out-of-sequence (OS) forwarding with delayed request for missing packets (RMP); we call it, in short, OSDRMP. It has the same reliability as PSFQ. In OSforwarding, a node can send a packet $p_{j}$ before sending one or more $p_{i}, i<j$. In particular, here a node $x$ can have the complete set of $n$ packets although none of its neighbors has the complete set; this is not possible in IS-forwarding. Also, a node $x$ can get all the packets quicker than in IS-forwarding. A node $x$ may have a missing packet $p_{i}$ for two reasons: $p_{i}$ has not been sent to $x$ or each transmission of $p_{i}$ to $x$ from its neighbors has failed to reach $x$. We use a minimum delay of $t_{r}$ time units from the time $p_{i}$ is detected missing at $x$ till $x$ can send the first RMP for $p_{i}$ to its neighbors; this allows enough time for $p_{i}$ to reach $x$ from some of its neighbors. The same delay $t_{r}$ is used between two successive RMPs from $x$ for a given $p_{i}$. This prevents too many unnecessary RMPs for $p_{i}$ in case none of the neighbors of $x$ currently has $p_{i}$.

\section{Key concepts}

\subsection{Acknowledgment vs. non-acknowledgment}

Both the acknowledgment $(A C K)$ and non-acknowledgment $(N A C K)$ based methods use three kinds of messages: (1) Transmission of a packet $p_{i}$, (2) Acknowledgment of a received packet $p_{i}$ in $A C K$ based method or Request for Missing Packet $p_{i}$ in $N A C K$-based method, and (3) Retransmission of a packet $p_{i}$. We denote them respectively by $T\left(p_{i}\right)$, $A C K\left(p_{i}\right), R M P\left(p_{i}\right)$, and $R T\left(p_{i}\right)$.

$A C K$-based method. Here, a node waits for an $\operatorname{ACK}\left(p_{i}\right)$ from the receiver for a minimum time period $t_{a}$ after sending a $T\left(p_{i}\right)$. If it does not receive an $A C K\left(p_{i}\right)$, then it sends an $R T\left(p_{i}\right)$ and waits again for a time $t_{a}$ for an $A C K\left(p_{i}\right)$, and the process continues till an $A C K\left(p_{i}\right)$ is received.

$N A C K$-based method. Here, with each $T\left(p_{i}\right)$ or $R T\left(p_{i}\right)$, we include the total number of packets $n$ so that a node can detect the missing packets once it receives a packet and send RMPs. The delay $t_{r}$ discussed in Section 1 applies to sending of RMPs. A node in this case does not wait for an $A C K\left(p_{i}\right)$ following a $T\left(p_{i}\right)$; instead, it sends an $R T\left(p_{i}\right)$ everytime it receives an $R M P\left(p_{i}\right)$. An $R M P\left(p_{i}\right)$ can be regarded as a negative acknowledgment. Unlike the $A C K$-based method, the NACK-based method cannot give $100 \%$ reliability if the successful transmission of a message has probability $<1$. 
Example 1. Figs. 2(a)-(b) show the timing diagrams for the transmission of $n=2$ packets $\left\{p_{0}, p_{1}\right\}$ from node 0 to node 1 in the network in Fig. 1(a) using $A C K$ and $N A C K$-based methods. We assume that only the first $T\left(p_{0}\right)$ is lost in both cases and all other messages are successful in the first attempt. The dashed lines in Fig. 2 show the lost transmissions. In Fig. 2(b), the receiving node 1 does not know the missing packet $p_{0}$ until it receives $p_{1}$. The example shows that the NACK-based method is better than the $A C K$-based method in terms of both the total delivery time and the total number of messages.

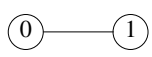

(a) A 2-node network.

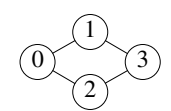

(b) A 4-node network.
Figure 1. Two simple networks.

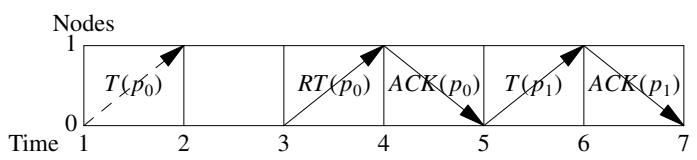

(a) $A C K$-based method with $t_{a}=1$.

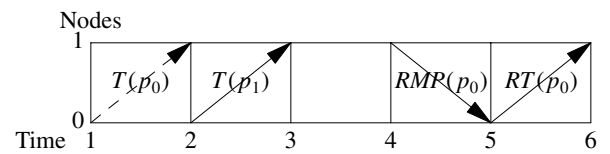

(b) NACK-based method with $t_{r}=1$.

Figure 2. An example of delivery of two packets $\left\{p_{0}, p_{1}\right\}$ in $A C K$ and $N A C K$-based methods.

\subsubsection{Analysis of message efficiency for $\left\{p_{0}, p_{1}\right\}$}

Assume as in above, that $p_{1}$ is successfully delivered to node 1 in one attempt. Let $P$ be the probability of successful transmission of a message from one node to its neighbor. Hence, the probability that $k$ transmissions are required for one message to be successfully sent from node 0 to node 1 is $P(1-\mathrm{P})^{k-1}$ and the expected number of transmissions is $1 / P$. Let $T_{S}$ and $T_{F}$ denote respectively a successful and a failed transmission of a packet $p_{i}$; we use similar abbreviations for successful and failed $R T, R M P$ and $A C K$.

In the $A C K$-based method, we have the following cases for the delivery of $p_{0}$ and $A C K\left(p_{0}\right)$ : (1) a $T_{S}\left(p_{0}\right)$ from node 0 to 1 followed by an $A C K_{S}\left(p_{0}\right)$ or an $A C K_{F}\left(p_{0}\right)$ from node 1 to node 0 , and (2) a $T_{F}\left(p_{0}\right)$ from node 0 to 1 (with no following $A C K\left(p_{0}\right)$ ). Except for the case of $T_{S}\left(p_{0}\right)$ followed by $A C K_{S}\left(p_{0}\right)$, there will be additional $R T\left(p_{0}\right) \mathrm{s}$ and $A C K\left(p_{0}\right) \mathrm{s}$. Let $E\left(p_{0}\right)=$ the expected number of messages for delivery of $p_{0}$ and $A C K\left(p_{0}\right)$. Then, $E\left(p_{0}\right)=P^{2} .2+$
$P(1-P)\left[2+E\left(p_{0}\right)\right]+(1-P)\left[1+E\left(p_{0}\right)\right]$, and hence $E\left(p_{0}\right)=(1+P) / P^{2}$. Since we assume node 0 delivers $p_{1}$ in one attempt, the expected number of messages $E\left(p_{1}\right)$ in delivering $p_{1}$ and $A C K\left(p_{1}\right)$ is $1 / P-1$ less than that for $p_{0}$, i.e., $E\left(p_{1}\right)=\left(1+P^{2}\right) / P^{2}$. Hence, the expected number of messages for delivery of $\left\{p_{0}, p_{1}\right\}$ and their acknowledgments is $\left(2+P+P^{2}\right) / P^{2}$.

In the $N A C K$-based method, after $T_{F}\left(p_{0}\right)$ and $T_{S}\left(p_{1}\right)$ from node 0 , node 1 detects that $p_{0}$ is missing and sends an $R M P\left(p_{0}\right)$. The computation of the expected number of messages $E^{\prime}\left(p_{0}\right)$ for the delivery of $p_{0}$ is slightly more complex now. With probability $P, p_{0}$ is delivered via the initial $T_{S}\left(p_{0}\right)$. If the first $T\left(p_{0}\right)$ fails, then this will be followed by an average $1 / P$ many $R M P\left(p_{0}\right)$ from node 1 to node 0 till the latter receives the request for $p_{0}$ and from that point on there will be an additional $E^{\prime}\left(p_{0}\right)$ many messages for the delivery of $p_{0}$. Thus, $E^{\prime}\left(p_{0}\right)=P .1+$ $(1-P)\left[1+1 / P+E^{\prime}\left(p_{0}\right)\right]$ and hence $E^{\prime}\left(p_{0}\right)=1 / P^{2}$. The expected number of messages for delivery of $\left\{p_{0}\right.$, $\left.p_{1}\right\}$ here is $1+1 / P^{2}$.

The $A C K$-based method therefore sends on the average $(1+P) / P^{2}$ extra messages. A similar analysis shows that the average number of messages received by nodes 0 and 1 is $2(1+P) / P$ for $A C K$-based method and $(1+P) / P$ for $N A C K$-based method. Thus, $A C K$ based receives $(1+P) / P$ extra messages.

\subsubsection{Analysis of message efficiency for $n \geq 2$}

In the case of $n \geq 2$ packets, the probability that at least one packet has been successfully delivered in the first attempt is $Q_{n}=1-(1-P)^{n}$ and assuming that this is the case the probability that $k \geq 1$ packets are delivered in the first attempt is $p(k)=$ $\frac{1}{Q_{n}}\left(\begin{array}{l}n \\ k\end{array}\right) p^{k}(1-p)^{n-k}$. The expected number of messages sent in $A C K$-based method for the delivery of all $n$ packets and their acknowledgments from node 0 to 1 is $\sum_{k \geq 1} p(k)\left[k . E\left(p_{1}\right)+(n-k)\left(1+E\left(p_{0}\right)\right)\right]$. For the $N A C K$-based method, the expected number of messages sent for the delivery of all $n$ packets is $\sum_{k \geq 1} p(k)\left[k+(n-k)\left(1+1 / P+E^{\prime}\left(p_{0}\right)\right)\right]$. This shows that the $A C K$-based method sends $n /\left(P Q_{n}\right)$ many extra messages, which can be very large when $P$ is small; it is close to $n$ when $P$ is close to 1 . We leave the analysis of the expected number of messages received for the general case to the reader.

\subsection{Message priority in OS-forwarding}

In OS, in general, the preferred priority order at a node among the message types is $\mathrm{T}>\mathrm{RT}>\mathrm{RMP}$. Since a node can send a packet $p_{j}$ without any 
constraint on $j$, it should send the packets as soon as possible so that a destination node $x$ may get different $p_{j}$ from its different neighbors at the earliest. The preferred priority order in IS-forwarding (as in PSFQ [1]) is RMP > RT > T; here, it is better for a node to request a missing packet $p_{i}$ because it cannot transmit $p_{j}, j>i$, before transmitting $p_{i}$. Unlike PSFQ, a node $x$ does not delay the sending of Ts and RTs in OS-forwarding.

Example 2. Figs. 3(a)-(b) show the minimum time and number of messages for delivering $n=2$ packets $\left\{p_{0}, p_{1}\right\}$ from node 0 to node 3 for the network in Fig. 1(b) using IS and OS. We assume that in both cases the only lost messages are $T\left(p_{0}\right)$ from node 0 to 2 and $T\left(p_{1}\right)$ from node 0 to 1 , as indicated by the dashed lines. Here, we use $t_{r}=0$ for simplicity. It shows how OS can achieve a better performance than IS.

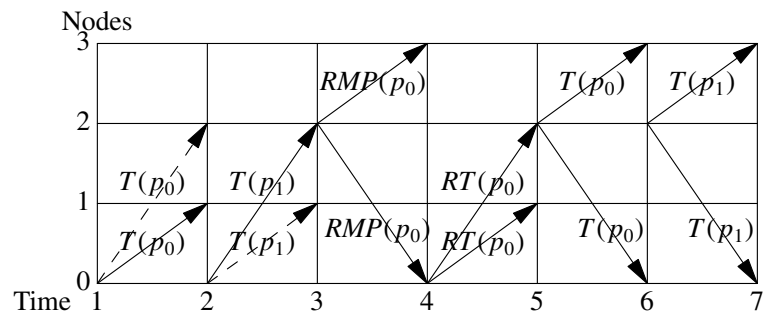

(a) The use of 7 time units and 12 messages in IS.

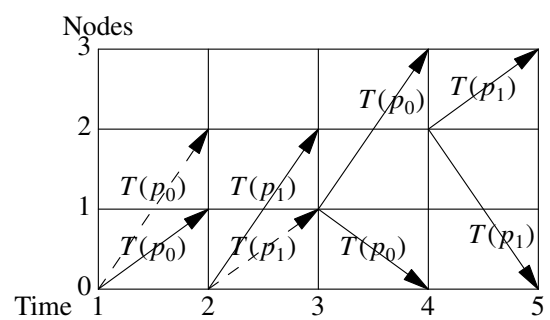

(b) The use of 5 time units and 8 messages in OS.

Figure 3. Comparison of IS and OS forwarding.

\subsection{NodeTTL and TTL in a message}

Each node $x$ which has received at least 1 message maintains a nodeTTL, which equals the maximum TTL (Time To Live) associated with those messages. Each message sent by $x$ to its neighbors includes $n$, nodeTTL $(x)-1$, the message type (T, RT, or RMP), and one of the following: (1) the packet sequence number $i$ and the data for $p_{i}$, for T and RT messages, and (2) the sequence number $i$ of a missing packet $p_{i}$, for RMP messages. For the source node $s$, nodeTTL $(s)$ is initialized to $d$ and it does not change. We refer to the component nodeTTL $(x)-1$ in a message associated with $p_{i}$ from $x$ as packetTTL $\left(p_{i}\right)$.
As a packet $p_{i}$ travels away from the source $s$ along various paths, the packetTTL $\left(p_{i}\right)$ associated with the T and RT messages for $p_{i}$ typically goes down by 1 with each step; it can also occasionally go up when it reaches a node that has previously received other messages along shorter paths. A node $x$ with current nodeTTL $(x)=0$ is considered a destination node, except that if at some later time nodeTTL $(x)$ becomes greater than 0 then from that point onwards $x$ remains permanently labeled as an intermediate node.

\section{The New Protocol}

\subsection{Local data at a node}

Each node $x$ with nodeTTL $(x) \geq 0$ has a Data Cache $\mathrm{DC}(x)=\left\{p_{i}: p_{i}\right.$ received by $\left.x\right\}$, a Transmission Queue $\mathrm{TQ}(x)$, a ReTransmission Queue $\operatorname{RTQ}(x)$ and a Request-for-Missing-Packet Queue $\operatorname{RMPQ}(x)$. At each node $x$, the following properties holds:

- $\mathrm{DC}(x)$ and $\mathrm{RMPQ}(x)$ are disjoint, $\mathrm{TQ}(x)$ and $\operatorname{RTQ}(x)$ are disjoint subsets of $\mathrm{DC}(x)$, and $|\mathrm{DC}(x) \cup \operatorname{RMPQ}(x)|=n$. None of $\mathrm{TQ}(x), \operatorname{RTQ}(x)$ and $\operatorname{RMPQ}(x)$ contains any duplicate item.

- The packets $p_{i}$ in TQ $(x)$ are ordered in the order of their arrival via Ts or RTs, and those in RTQ $(x)$ are ordered in the order of their arrival via RMPs. $\operatorname{RMPQ}(x)$ is maintained as a circular list, ordered by the packet sequence numbers.

Example 3. Fig. 4 shows $\operatorname{DC}(x)$ of a node $x$ for $n=$ 9 and the arrivalTime of each $p_{i}$ in DC; in particular, the packets arrived in the order $p_{1}, p_{4}, p_{5}, p_{0}$, and $p_{7}$. The packetTTL $\left(p_{i}\right)$ is shown in parentheses next to each $p_{i}$ in DC. Each of TQ $(x), \operatorname{RTQ}(x)$ and $\operatorname{RMPQ}(x)$ points to the first packet in the corresponding queue and the arrows from one packet to another show the sequence of packets in the queue. We also show the updates to nodeTTL $(x)$ as each $p_{i} \in \mathrm{DC}$ is received.

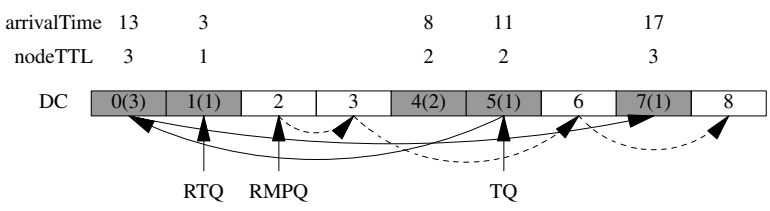

Figure 4. Illustration of DC, TQ, RTQ, RMPQ, and updating of nodeTTL at a node.

\subsection{Message processing at a node}

In one time unit, a node $x$ can do one of the following: (1) receive a message from one of its neighbors and process it, (2) send a message to each of its neighbors, and (3) remain idle. 


\subsubsection{Nodes selected for message transmission}

Two nodes can send a message at time $t$ only if the hop-distance between them $\geq 3$. The set of candidate nodes that are eligible to send a message at time $t$ is given by $S^{\prime}(t)=\{x: \operatorname{TQ}(x) \cup \operatorname{RTQ}(x) \neq \varnothing$ or $\operatorname{RMPQ}(x) \neq \varnothing$ and $x$ can send $\operatorname{RMP}\left(p_{i}\right)$ at time $t$ for some $\left.p_{i} \in \operatorname{RMPQ}(x)\right\}$. We can exclude a node $x$ with $\operatorname{nodeTTL}(x)=0$ from $S^{\prime}(t)$ for sending $T$ and $R T$ messages; this tends to reduce the number of messages slightly without significantly affecting the total delivery time in spite of the fact two destination nodes may be adjacent to each other and that a nodeTTL $(x)$ may become positive at a future time.

Any protocol that prevents message collision at a node from two or more of its neighbors may be used to decide the group of nodes $S(t) \subseteq S^{\prime}(t)$ that sends messages at time $t$. In our simulation (see Section 4), we select $S(t)$ as a random maximal subset of $S^{\prime}(t)$ such that no two nodes in $S(t)$ are within hop-distance 2 of each other. A node in $S(t)$ transmits a message from one of its TQ, RTQ or RMPQ in that priority order. A node $x$ sends $\operatorname{RMP}\left(p_{i}\right)$ for the first $p_{i} \in \operatorname{RMPQ}(x)$ which satisfies $t_{r}$ delay requirement. For each $p_{i}$, a node can send $\mathrm{T}\left(p_{i}\right)$ only once but it can send $\operatorname{RMP}\left(p_{i}\right)$ and $\operatorname{RT}\left(p_{i}\right)$ multiple times. At time $t=0$, $S^{\prime}(0)=S(0)=\{s\}$. (Note that we cannot give priority to certain nodes $x$, say, based on nodeTTL $(x)>0$ for selection in $S(t)$ since this would require a complex protocol in itself for the determination of $S(t)$.)

\subsubsection{Processing of input}

In addition to the update of nodeTTL $(x)$, the datacache and various queues at $x$ are updated as follows when a node $x$ receives an input. A node $x$ processes an input message if and only if it is the first message to $x$ with packetTTL $\geq 0$ or nodeTTL $(x) \geq 0$. If the first message with packetTTL $\geq 0$ is a packet $p_{i}$ (via $T\left(p_{i}\right)$ or $\left.R T\left(p_{i}\right)\right), x$ initializes $\mathrm{DC}(x)=\left\{p_{i}\right\}=\mathrm{TQ}(x)$, $\operatorname{RTQ}(x)=\varnothing$, and $\operatorname{RMPQ}(x)=\left\{p_{j}: j \neq i\right.$ and $j \leq$ $n-1\}$. If the first message with packetTTL $\geq 0$ is an $\operatorname{RMP}\left(p_{i}\right), x$ initializes $\operatorname{RMPQ}(x)$ with $p_{0}$ and $\mathrm{DC}(x)=$ $\mathrm{TQ}(x)=\operatorname{RTQ}(x)=\varnothing$. When nodeTTL $(x) \geq 0$, if the input is $\mathrm{T}\left(p_{i}\right)$ or $\mathrm{RT}\left(p_{i}\right)$ and $p_{i} \notin \mathrm{DC}(x)$, then $p_{i}$ is added to both $\operatorname{DC}(x)$ and $\mathrm{TQ}(x)$ and is removed from $\operatorname{RMPQ}(x)$, if necessary, $\operatorname{RMPQ}(x)$ is updated with all $p_{j}$ where $p_{j} \notin \mathrm{DC}(x) \cup \mathrm{RMPQ}(x)$; if $p_{i} \in \mathrm{DC}(x)$ then no other updates take place. If the input is $\operatorname{RMP}\left(p_{i}\right)$ then $p_{i}$ is added to $\operatorname{RTQ}(x)$ provided $p_{i} \in \mathrm{DC}(x)$ and $p_{i} \notin \mathrm{TQ}(x) \cup \operatorname{RTQ}(x)$; otherwise, no other updates occur.

\section{Simulation}

We simulate the OSDRMP and PSFQ protocols on the network in Fig. 5 for $n=10$ for different source node $s$ to evaluate their performance based on the total delivery time (delivTime) and the total number of messages sent and received (numMess). When a node $x$ sends a message ( $T, R T$, or $R M P$ ) to its neighbors, we count this as one message in numMess irrespective of the number of neighbors of $x$ in order to reflect its impact on the energy consumption at $x$; each message received by $x$ is also counted as one message in numMess. A simulation run is considered successful if at least one destination node $x$ (i.e., $\operatorname{nodeTTL}(x)=$ 0 ) is found and each node $x$ with nodeTTL $(x)=0$ has $|\mathrm{DC}(x)|=n$ and $\mathrm{TQ}(x)=\mathrm{RTQ}(x)=\varnothing$; the condition $\mathrm{TQ}(x)=\mathrm{RTQ}(x)=\varnothing$ reduces the probability that there are other potential destinations nodes that have not received any $p_{i}$ yet.

We consider two variations of RMPs described below. The second one applies only to OSDRMP.

(1) Restricted RMP (rRMP): Let $m_{x}$ be the highest packet sequence number in $\mathrm{DC}(x)$ of a node $x$; we let $m_{x}=-1$ if $\operatorname{DC}(x)=\varnothing$. Here, $x$ can send an $\operatorname{RMP}\left(p_{j}\right)$ at time $t$ only if $j<m_{x}$; the other restrictions that $p_{j} \in \operatorname{RMPQ}(x)$ and $x$ satisfies the delay requirement $t_{r}$ still apply. If there is no $p_{j} \in \operatorname{RMPQ}(x)$ with $j<m_{x}$ and $\operatorname{RMPQ}(x) \neq \varnothing$ (i.e., $m_{x}<n-1$ ), then $x$ can send $\operatorname{RMP}\left(p_{j}\right)$ for $j$ $=m_{x}+1$.

(2) Unrestricted RMP (urRMP): This is the normal case without the restrictions in rRMP.

We compute the minimum delay $t_{r}$ for a node $x$ according to $t_{r}=$ degree $(x) \times \mathrm{RMPdelayFactor}$, where degree $(x)$ is the number of neighbors of $x$ and RMPdelayFactor is a constant $=\delta$. A time point $t$ is considered an idleTime if $S^{\prime}(t)=\varnothing$.

We show below the results of simulation of OSDRMP (both rRMP and urRMP) and PSFQ for the network in Fig. 5 for $P=0.3,0.6$ and 0.9 using different RMPdelayFactor. We also show the results for the same network using $s=0$ and TTL $=14$.

\subsection{Simulation results for $s=\mathbf{4 0}$ and $\mathrm{TTL}=5$}

Figs. 6(a)-(f) show the variation of delivTime and numMess with RMPdelayFactor for OSDRMP(rRMP and urRMP) and PSFQ at $P=0.3,0.6$ and $0.9, s=40$ and TTL $=5$. For higher $P$, we use a larger range of RMPdelayFactor to better represent the trend of variation of delivTime and numMess.

Both versions of OSDRMP perform better than PSFQ in terms of both delivTime and numMess when $P=0.6$ and 0.9. For $P=0.3$, numMess for PSFQ are 


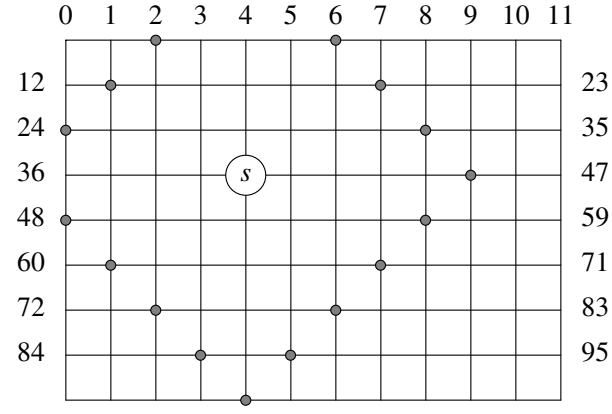

96979899100101102103104105106107

Figure 5. A $12 \times 9$ grid network; the intended destination nodes are marked for $s=40$ and TTL $=5$.

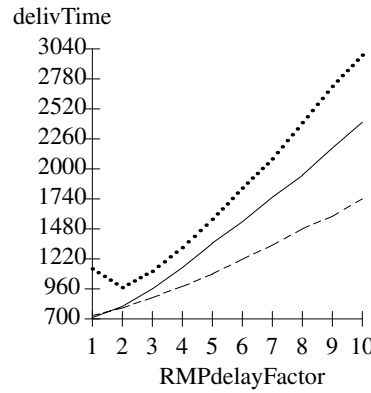

(a) $P: 0.3$

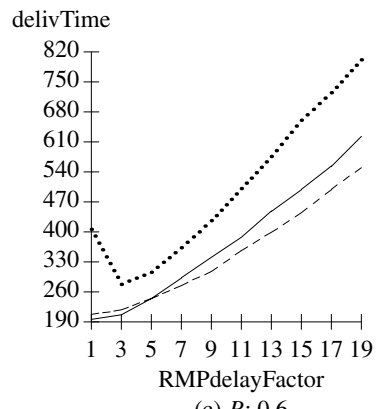

(c) $P: 0.6$

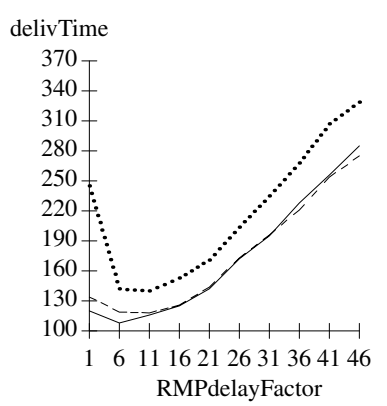

(e) $P: 0.9$

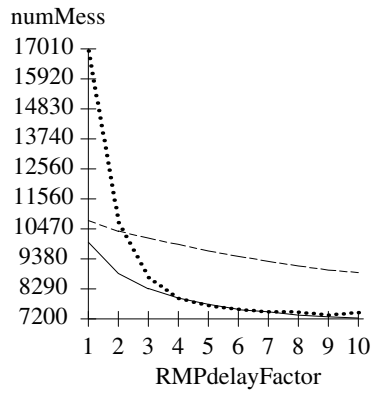

(b) $P: 0.3$

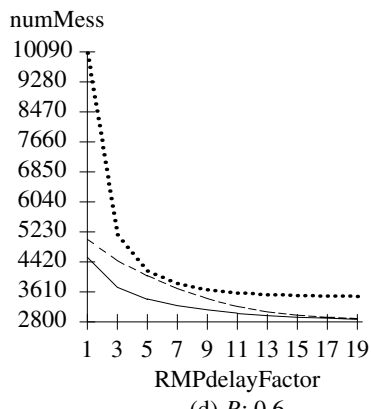

(d) $P: 0.6$

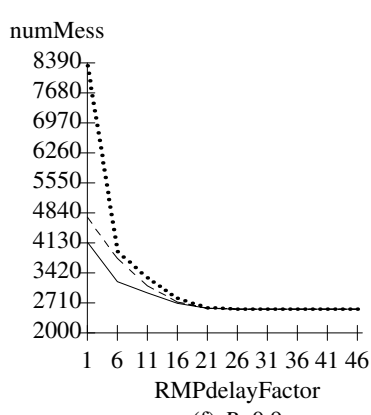

(f) $P: 0.9$
OSDRMP(rRMP) -----OSDRMP(urRMP) ........PSFQ

Figure 6. Comparison of OSDRMP and PSFQ for the network in Fig. 5.

initially higher than that for OSDRMP(urRMP) but as RMPdelayFactor increases, PSFQ performs better than OSDRMP(urRMP) but this comes only with a very large increase in delivery time.

\subsubsection{Analysis: delivTime vs. RMPdelayFactor}

Consider Figs. 6(a), (c), and (e) for different $P$ s. In each case, the delivTime of PSFQ first decreases and then increases as we increase the RMPdelayFactor $=\delta$. When $\delta$ is small, the higher priority of RMP in PSFQ does not give enough opportunities for a node $x$ to send Ts and RTs to its neighbors, which can result in more RMPs for some of the neighbors of $x$. Even if $\operatorname{RMPQ}(x)=\varnothing$, the frequent competition for RMP from the neighbors of $x$ decreases $x$ being selected for $\mathrm{T}$ or RT, and this too can lead to more RMPs from the neighbors. The result is higher delivTime. On the other hand, when $\delta$ is increased beyond a certain value it results in increased idleTime and this increases the delivTime. For a given $\delta$, the increase in $P$ causes fewer RMPs and this decreases the delivTime. Note that the minimum delivTime occurs at a higher $\delta$ as $P$ increases.

In OSDRMP, T and RT have priority. So for low $\delta$, the delay of Ts and RTs due to frequent transmission of RMPs is very low and hence initial increase in $\delta$ benefits OSDRMP's delivTime much less than that of PSFQ. Like PSFQ, at higher $P$, increase in $\delta$ benefits propagation of successful Ts and RTs through the network and hence, idleTime contributes to increase in delivTime at higher $\delta$ in comparison to that at lower $P$.

For $P=0.3$, there are a large number of unsuccessfully transmitted RMPs and hence the delay between RMPs can be effectively utilized by sending more RMPs as in case of OSDRMP(urRMP). But as $P$ increases, more RMPs are successfully transmitted and hence it is good to wait for corresponding RTs instead of sending more RMPs. So the difference in delivTime between OSDRMP with rRMP and with urRMP decreases as $P$ increases, and for $P=0.9$ the two versions of OSDRMP have similar delivTime.

\subsubsection{Analysis: numMess vs. RMPdelayFactor}

For a low $\delta$, highest priority to RMP and frequent transmission of RMPs in PSFQ with delayed Ts and RTs leading to more RMPs, add up to a high numMess for all $P$. With increase in $\delta$, Ts and RTs have enough time to reach nodes thus reducing the number of RMPs and the numMess for PSFQ. For low $\delta$, both versions of OSDRMP perform better than PSFQ as T and RT have higher priority in OSDRMP. For $P=0.3$, unsuccessful transmissions coupled with urRMP and nodes requesting packets which none of their neighbors have (due to OS) contribute to the high numMess for OSDRMP(urRMP) compared to that for PSFQ where there is rRMP and if a node requests a packet $p_{i}, i<m$ 
( $m$ is the highest packet sequence number in the node's DC), at least one of its neighbors has it. For large $\delta$ and small $P=0.3$, the rRMP in OSDRMP(rRMP) eliminates any potential disadvantage of OS and thus the numMess becomes almost equal to that of PSFQ. For $P=0.6$ and 0.9 , OS combined with successful transmissions result in destinations receiving $n$ packets much faster than PSFQ reducing RMPs and RTs and duplicate Ts to the same node and hence, the numMess required for both versions of OSDRMP with increasing $\delta$ is almost equal and at times better than that required for PSFQ.

There is a $\delta$ at each $P$ for each protocol after which the numMess will not change with increase in $\delta$. The reason is that all Ts and RTs have been transmitted and it is not time for an RMP from any node. Increase in $\delta$ does not benefit this situation and only contributes to idleTime. The $\delta$ after which numMess becomes constant increases with increase in $P$ for both OSDRMP(rRMP) and PSFQ. This is because there are more successful transmissions at higher $P$ and increase in $\delta$ helps in propagation of successful transmissions. In case of OSDRMP(urRMP), urRMP, unsuccessful transmissions and requests from nodes for packets not present at any of their neighbors at lower $P(=0.3)$ result in a large number of RMPs and the $\delta$ after which numMess do not change is large compared to that at higher $P$. However as $P(=0.6$ and 0.9$)$ increases, the behavior of OSDRMP(UrRMP) is similar to that of OSDRMP(rRMP).

\subsection{Simulation results for $s=0$ and TTL $=14$}

Figs. 7(a)-(f) show the variations of delivTime and numMess with $\delta$ for OSDRMP(rRMP and urRMP) and PSFQ for $P=0.3,0.6$ and 0.9 . Here, the same conclusions as those in Fig. 6 hold, except that there are more messages in Fig. 7 (because of larger TTL) which also causes the increase in idleTime start at a higher $\delta$.

\section{Conclusions}

Our simulations demonstrate the superiority of OSDRMP over PSFQ in terms of both the total number of messages and the total delivery time. Indeed, the improvements depend on the network structure and the probability of a successful message transmission. Our future work will consider different priority orders at different nodes based on their nodeTTL (say); it is also possible that nodeTTL can be used more effectively in responding to requests for a packet.

\section{References}

[1] C.-Y.Wan, A.T.Campbell and L. Krishnamurthy, "PSFQ: A reliable transport protocol for wireless sensor networks",
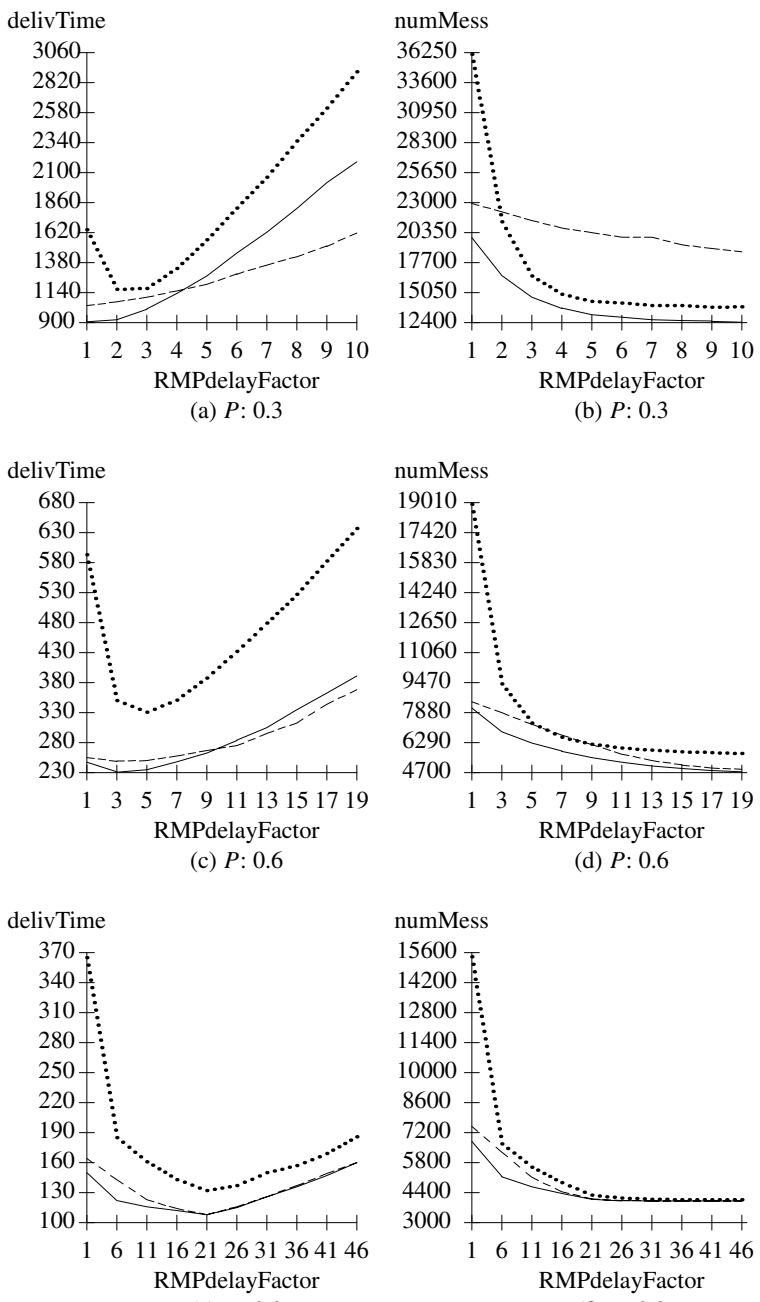

(e) $P: 0.9$

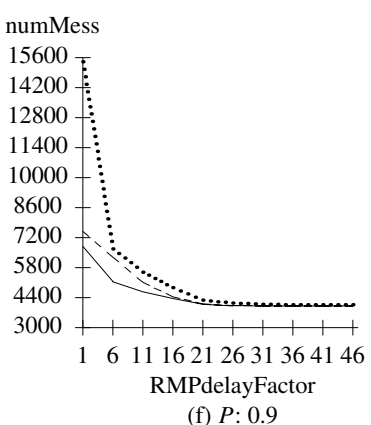

OSDRMP(rRMP) ---- OSDRMP(urRMP) ….....PSFQ

Figure 7. Comparison of OSDRMP and PSFQ for the network in Fig. 5 with $s=0$ and TTL $=14$.

in Proc. $1^{\text {st }}$ Intl. Workshop on Wireless Sensor Networks and Appl., GA, 2002.

[2] F. Stann and J. Heidemann, "RMST: Reliable data transport in sensor networks", in Proc. $1^{\text {st }}$ IEEE Intl. Workshop on Sensor Network Protocols and Appl., Alaska, May 2003.

[3] Y. Sankarsubramaniam, O. Akan, I. Akyildiz, "ESRT: Event-to-sink reliable transport in wireless sensor networks" in Proc. ACM Mobihoc 2003, Maryland, June 2003.

[4] A. DeSimone, M.C. Chuah and O.-C. Yue, "Throughput performance of transport-layer protocols over wireless LANs", Proc. of IEEE Globecom, Dec 1993.

[5] A. Woo, T. Tong, and D. Culler, "Taming the underlying challenges of reliable multihop routing in sensor networks", ACM Sensys, November 2003.

[6] R. Iyer and L. Kleinrock, "QoS control for sensor networks", IEEE Intl. Conf. on Comm., May 2003. 